\title{
Appetite testing in HIV-infected African adults recovering from malnutrition and given antiretroviral therapy
}

\author{
Andrea M Rehman' ${ }^{1}$ Susannah Woodd', Molly Chisenga ${ }^{2}$, Joshua Siame², Gemma \\ Sampson ${ }^{1}$, George PrayGod ${ }^{3}$, John R Koethe ${ }^{4}$, Paul Kelly ${ }^{2}$ and Suzanne Filteau ${ }^{1, *}$ \\ ${ }^{1}$ Faculty of Epidemiology and Population Health, London School of Hygiene and Tropical Medicine, Keppel Street, \\ London WCIE 7HT, UK: ${ }^{2}$ University Teaching Hospital, Lusaka, Zambia: ${ }^{3}$ National Institute of Medical Research, \\ Mwanza, Tanzania: ${ }^{4}$ Vanderbilt University School of Medicine, Nashville, TN, USA
}

Submitted 14 October 2013: Final revision received 25 February 2014: Accepted 27 March 2014: First published online 1 May 2014

\begin{abstract}
Objective: The Nutritional Support for Africans Starting Antiretroviral Therapy (NUSTART) trial was designed to determine whether nutritional support for malnourished HIV-infected adults starting antiretroviral therapy (ART) can improve early survival. Appetite is related to health outcomes in this population, but the optimal appetite metric for field use is uncertain. We evaluated two measures of appetite with the goal of improving understanding and treatment of malnutrition in HIV-infected adults.

Design: Longitudinal cohort study embedded in a clinical trial of vitamin and mineral-fortified, $v$. unfortified, lipid-based nutritional supplements.

Setting: HIV clinics in Mwanza, Tanzania and Lusaka, Zambia.

Subjects: Malnourished (BMI $<18.5 \mathrm{~kg} / \mathrm{m}^{2}$ ) HIV-infected adults starting ART.

Results: Appetite measurements, by short questionnaire and by weight of maize porridge consumed in a standardized test, were compared across time and correlated with changes in weight. Appetite questionnaire scores, from polychoric correlation, and porridge test results were normally distributed for Tanzanians ( $n$ 187) but clustered and unreliable for Zambians ( $n$ 297). Among Tanzanian patients, the appetite score increased rapidly from referral for ART, plateaued at the start of ART and then increased slowly during the 12-week follow-up. Change in appetite questionnaire score, but not porridge test, correlated with weight change in the corresponding two-week intervals $(P=0.002)$ or over the whole study $(P=0.05)$ but a point estimate of hunger did not predict weight change $(P=0 \cdot 4)$.

Conclusions: In Tanzania change in appetite score correlated with weight change, but single point measurements did not. Appetite increases several weeks after the start of ART, which may be an appropriate time for nutritional interventions for malnourished HIV-infected adults.
\end{abstract}

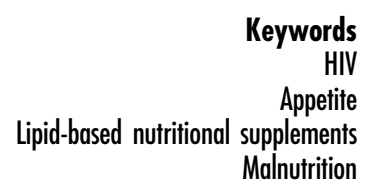

The roll out of antiretroviral therapy (ART) in Africa over the past decade has saved many lives but there remains a worryingly high risk of mortality in the first few months after starting $\mathrm{ART}^{(1)}$. Malnutrition, as indicated by $\mathrm{BMI}<18.5 \mathrm{~kg} / \mathrm{m}^{2}$ in adults, is an independent risk factor for this early mortality ${ }^{(1)}$. However, even under controlled research conditions, food supplements may not have efficacy for decreasing mortality ${ }^{(2,3)}$. Well-established treatment regimens for severe malnutrition in young children $^{(4)}$ use a two-stage protocol in which metabolic changes resulting from malnutrition and concurrent infections are stabilized before larger amounts of energy are provided for weight regain. Therefore, in our Nutritional Support for Africans Starting Antiretroviral
Therapy (NUSTART) trial we wished to test whether a similar two-stage protocol could improve survival of malnourished adults starting ART.

When treating severely malnourished young children, a key indicator of when a child is ready to move from the first stage of metabolic stabilization to the second stage of weight gain is return of appetite ${ }^{(4)}$, although this may not always be observed in HIV-infected malnourished children ${ }^{(5)}$. Appetite is also of concern in malnourished patients starting ART because too rapid an increase in appetite may result in a harmful refeeding syndrome ${ }^{(6)}$. A third reason to study appetite of HIV-infected patients is that some food-insecure patients are reported not to adhere well to ART because of experiences or fears that 
the ART drugs increase hunger ${ }^{(7,8)}$. We wished to develop a measure of appetite that would both assist in understanding appetite interactions with nutritional metabolism during ART and aid clinical management of patients.

Published tools for measuring appetite or hunger may not be applicable to a population of underweight, seriously ill African adults starting ART; for example, tools may have been validated for very different populations, such as obese subjects on low-energy diets or young African children fed by their mothers ${ }^{(9)}$. Furthermore, the concept of 'hunger' among low-income populations is often used as overlapping with 'food security' ${ }^{\text {(10) }}$, which is only one component of appetite and food intake of interest in the present work. We chose to use two appetite tests: (i) an adaptation of a short questionnaire previously used successfully for elderly American adults ${ }^{(11)}$, incorporating a food security question; and (ii) a test of intake of a standard porridge, similar to tests validated in young children in Benin ${ }^{(9)}$ and also used for young South African children ${ }^{(12)}$ and Kenyan schoolchildren ${ }^{(13)}$. We compared these two tests with body weight changes over time, which we considered the most robust estimate of energy intake of adults recovering from malnutrition.

\section{Methods}

The study was embedded in the NUSTART trial (trial registration \# PACTR201106000300631) conducted in Mwanza, Tanzania and Lusaka, Zambia from August 2011 and used data entered to the end of June 2013 although the main NUSTART trial continued to recruit. HIV-infected male and female participants were recruited to the NUSTART trial if they: (i) were at least 18 years old; (ii) were ART-naïve except for standard regimens to prevent maternal-to-child HIV transmission; (iii) had $\mathrm{BMI}<18.5 \mathrm{~kg} / \mathrm{m}^{2}$; (iv) required ART as determined by CD 4 count $<350$ cells/ $\mu$ l or WHO stage 3 or 4 disease; (v) were willing to undertake intensive follow-up in the study clinic; and (vi) provided written or thumbprint, informed consent. NUSTART is an individually randomized, double-blind, placebo-controlled trial of vitamin and mineral-fortified lipid-based nutritional supplements (LNS; prepared by Nutriset, Malauney, France) for malnourished patients starting ART, with mortality from recruitment to 12 weeks after starting ART as the primary outcome. Participants randomized to the intervention arm were provided with a low-kilojoule (30 g) LNS rich in all essential micronutrients, except $\mathrm{Fe}$, and electrolytes (LNS-VM) from recruitment until 2 weeks after starting ART. ART was provided by routine health services at both sites. The decision when to start ART is made partly on clinical grounds, e.g. after stabilization of any associated medical conditions, and partly on patient readiness to begin lifelong ART. Following the low-kilojoule supplement, participants received a high-kilojoule $(250 \mathrm{~g}$ or $5690 \mathrm{~kJ} / \mathrm{d}(1360 \mathrm{kcal} / \mathrm{d}))$ LNS containing the same vitamins and minerals, plus
Fe, during weeks 2-6 of ART. No supplement was given from weeks 6 to 12 of ART. Placebo LNS preparations were identical in total kilojoules, consistency, product quantity, taste and smell but lacked the vitamin and mineral fortificant. The two-step regimen was designed to mimic standard protocols for management of severe malnutrition in young children in that there was a stabilization period where vitamins and minerals were provided and infections were treated before energy was provided in order to promote weight gain ${ }^{(4)}$. The trial is ongoing and the investigators remain blind, but the intervention is not the subject of the present article.

Patients came to the clinic weekly until the start of ART for medical examination, questionnaires, anthropometry, blood sampling for electrolytes, appetite testing and to pick up their next lot of supplements. Patients also came to the clinic at weeks 1, 2, 4, 6, 8 and 12 after starting ART. Height was measured at recruitment and weight at each visit using a stadiometer fixed to the wall and a digital balance, respectively; measurements were taken in triplicate and the median used in analyses. Technical errors of measurement were assessed using standard methods and were within acceptable limits for height and weight at both sites ${ }^{(14)}$.

The appetite questionnaire administered at each visit contained four questions with graded levels of response (Table 1). We also used an appetite test that was similar to an ad libitum maize porridge consumption test previously validated against $3 \mathrm{~d}$ weighed intakes among young children in Benin ${ }^{(9)}$ and also used in young South African children $^{(12)}$ and school-aged Kenyan children ${ }^{(13)}$. For the appetite test, patients were asked to come to the clinic having fasted since the previous evening; a questionnaire determined what, if anything, they had eaten since the night before. A standard porridge was prepared daily using 1.1 litres of maize meal and 4 litres of water; this was boiled for $30 \mathrm{~min}$ and kept warm until eaten; salt or sugar was added to taste. Patients were provided with a bowl containing about $250 \mathrm{~g}$; the bowl and porridge together were weighed to $10 \mathrm{~g}$ precision before giving it to the participants and after the participants had eaten as much as they wished in order to calculate amount consumed by difference. Participants could also request additional bowls. Due to concern regarding the effect of cultural norms on patient requests for additional bowls in Zambia, we increased the first bowl weight to $400 \mathrm{~g}$ between 19 June and 20 July 2012.

\section{Statistics and data management}

Data were double entered into OpenClinica in Zambia and MySQL in Tanzania. Analysis and data cleaning were carried out in the Stata statistical software package version 12.1. Analysis was restricted to patients who completed follow-up to 12 weeks after ART as those who died or withdrew would be expected to have different profiles for 
Table 1 Appetite questionnaire and baseline responses; Nutritional Support for Africans Starting Antiretroviral Therapy (NUSTART) trial

\begin{tabular}{|c|c|c|c|c|c|}
\hline & & \multicolumn{2}{|c|}{$\begin{array}{l}\text { Zambians } \\
\text { (n 297) }\end{array}$} & \multicolumn{2}{|c|}{$\begin{array}{c}\text { Tanzanians } \\
(n 182)^{\star}\end{array}$} \\
\hline & & $n$ & $\%$ & $n$ & $\%$ \\
\hline \multirow[t]{6}{*}{ \#1 } & How would you describe your appetite over the past $7 d$ ? & & & & \\
\hline & 1. Excellent & 10 & 3 & 13 & 7 \\
\hline & 2. Good & 109 & 37 & 43 & 24 \\
\hline & 3. Usual & 63 & 21 & 55 & 30 \\
\hline & 4. Poor & 111 & 37 & 60 & 33 \\
\hline & 5. Very poor, not wanting to eat very much at all & 4 & 1 & 11 & 6 \\
\hline \multirow[t]{5}{*}{ \#2 } & Have you been hungry in the past $7 d$ ? & & & & \\
\hline & 1. Not hungry at all, don't want to eat or can't finish my plate of food & 86 & 29 & 18 & 10 \\
\hline & 2. Averagely hungry & 98 & 33 & 61 & 34 \\
\hline & 3. Moderately hungry so needed more than 3 meals per day & 104 & 35 & 47 & 26 \\
\hline & 4. Very hungry often (e.g. hunger interfering with sleep at night or daily activities) & 9 & 3 & 56 & 31 \\
\hline \multirow[t]{3}{*}{ \#3 } & Are you usually able to afford enough food to satisfy your hunger? & & & & \\
\hline & 1. Yes & 252 & 85 & 131 & 72 \\
\hline & 2. No & 45 & 15 & 51 & 28 \\
\hline \multirow[t]{6}{*}{ \#4 } & In the past $7 \mathrm{~d}$, how has food usually tasted? & & & & \\
\hline & 1. Very bad & 32 & 11 & 9 & 5 \\
\hline & 2. Bad & 56 & 19 & 47 & 26 \\
\hline & 3. Average & 76 & 26 & 52 & 29 \\
\hline & 4. Good & 126 & 42 & 70 & 38 \\
\hline & 5. Very good & 7 & 2 & 4 & 2 \\
\hline
\end{tabular}

*Five of the 187 did not answer the questionnaire.

weight change. Patients who delayed starting ART and remained on the low-kilojoule supplement for 10 weeks or more were also excluded from analysis. The four appetite questions at each patient visit were combined into single standardized scores by polychoric correlation ${ }^{(15)}$ with lower scores representing lower appetite.

Changes in appetite score and changes in weight over time were assessed with regression using restricted cubic splines with five $\operatorname{knots}^{(16)}$. The time axis was centred around the date of starting ART; hence visits pre-ART had negative values. The analysis was stratified by the number of weeks spent in the pre-ART phase as this influenced the shape of the splines. To determine the association of appetite score and test results with weight gain, considered a measure of energy intake, we focused on four key time points: recruitment, start of ART, 2 weeks after starting ART at which point the kilojoules provided in the intervention increased and 6 weeks after starting ART at which point the intervention ceased. Weight change during the following two weeks after appetite assessment was selected to be long enough to permit detectable weight change but short enough to limit the amount of change in clinical condition. Mixed-effects linear regression was used to compare change in appetite score (divided into a decrease, change $\leq-0.25$; little change, $-0.25<$ change $<$ 0.25 ; and an increase, change $\geq 0.25$ ) with change in weight during the subsequent two weeks after the four key time points; the regression model controlled for sex and repeated measures of individuals. Additional potential confounders that were considered a priori were baseline BMI (divided into $<16 \cdot 0,16 \cdot 0-16.99,17 \cdot 0-18.49 \mathrm{~kg} / \mathrm{m}^{2}$ ), baseline CD4 count (divided into <50, 50-99, 100-199, $\geq 200$ cells/ $\mu$ l), oedema at baseline, tuberculosis status preART, socio-economic status (measured by principal component analysis ${ }^{(17)}$ within each site since sites differed and categorized into quintiles) and, for the appetite test, whether or not the patient was fasting before the test. We also present the relationship between appetite and overall change in weight from recruitment to 12 weeks of ART. Finally, we determined whether a single question about hunger, $\# 2$ on the questionnaire, at a single time point could be used to predict subsequent two-week weight gain since this might prove clinically useful.

\section{Etbics}

The NUSTART trial was conducted according to guidelines laid down in the Declaration of Helsinki and all procedures were approved by the ethics committees of the London School of Hygiene and Tropical Medicine, the University of Zambia and the National Institute for Medical Research, Tanzania. Participants provided written or thumbprint informed consent. Medical care for participants was provided as needed by study clinicians or the local referral hospitals.

\section{Results}

\section{Patient characteristics and weight changes}

Figure 1 shows the patients included in the analyses and Table 2 provides their sociodemographic and clinical characteristics at recruitment. Tanzanian patients were older and had lower CD 4 count at screening, were less 


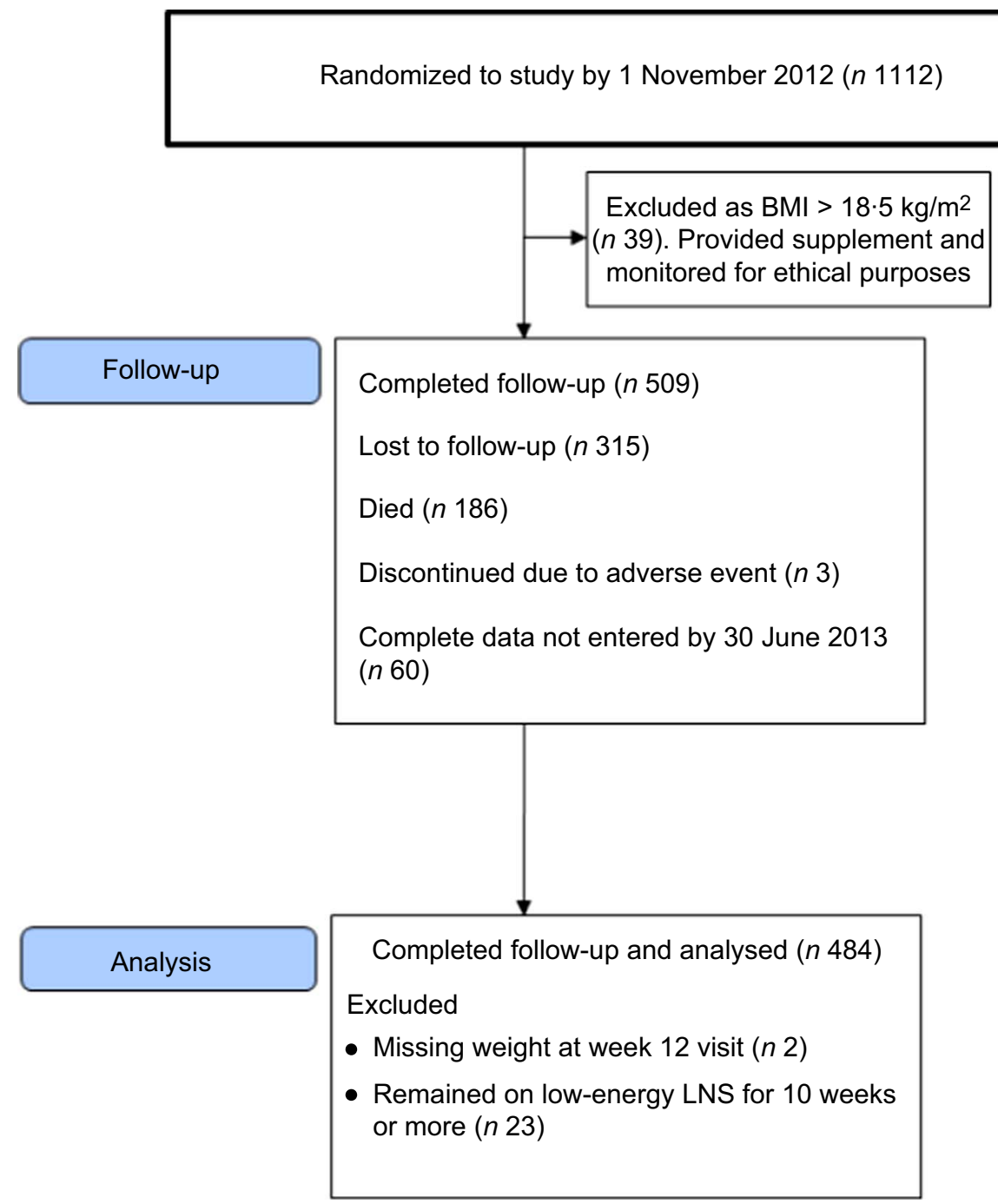

Fig. 1 (colour online) Flow diagram of patients included in the Nutritional Support for Africans Starting Antiretroviral Therapy (NUSTART) trial (LNS, lipid-based nutritional supplement)

educated, were more likely to be self-employed and were less likely to be married than Zambian patients. The median time between recruitment and starting ART was $19 \mathrm{~d}$ (interquartile range: $15-26 \mathrm{~d}$ ). Figure 2 shows weight over time in the study for male and female participants starting ART between 14 and $20 \mathrm{~d}$, both the median and the modal (most frequent) week post-recruitment in the trial population; curves were similar, although more variable due to smaller sample sizes, for subgroups with other durations of pre-ART phase and the 14-20 d subgroup was chosen for simplicity of presentation. From recruitment to week 12 after starting ART, Tanzanian men had gained a mean of $6.68 \mathrm{~kg}$ (95\% CI 5.48, $7.87 \mathrm{~kg}, n$ 77) since recruitment, Tanzanian women a mean of $5.96 \mathrm{~kg}(95 \% \mathrm{CI}$ $5.24,6.69 \mathrm{~kg}, n$ 110), Zambian men a mean of $3.44 \mathrm{~kg}$ (95\% CI $2.63,4.24 \mathrm{~kg}, n$ 149) and Zambian women a mean of $4.16 \mathrm{~kg}$ (95\% CI $3.45,4.87 \mathrm{~kg}, n$ 148). These increases represent a significant difference by site $(P<0 \cdot 01)$ but no difference between males and females.
About $40 \%$ of the weight increase occurred during the four weeks the patients were taking the high-kilojoule supplement and about $35 \%$ occurred prior to starting the high-kilojoule supplement.

\section{Appetite results}

At recruitment, just over a third of patients reported having low appetite (Table 1) and there were differences in reporting patterns between Tanzanians and Zambians. Among Tanzanian patients the appetite score was approximately normally distributed. Among Zambian patients the scores were clustered around certain values: $44 \%$ of all responses were good appetite, average or moderately hungry, could afford food and food tasted good; a further $10 \%$ of responses were usual appetite, average hunger, could afford food and food tasted average. Because of the clustered nature of responses it was not possible to generate an appetite score for Zambian 
Table 2 Baseline characteristics of the study population; Nutritional Support for Africans Starting Antiretroviral Therapy (NUSTART) trial

\begin{tabular}{|c|c|c|c|c|}
\hline & \multicolumn{2}{|c|}{ Zambians } & \multicolumn{2}{|c|}{ Tanzanians } \\
\hline & $n$ or Mean & $\%$ or SD & $n$ or Mean & $\%$ or SD \\
\hline Population, $n$ and $\%$ & 297 & 61 & 187 & 39 \\
\hline Age (years), mean and SD & 34.7 & 9.5 & $38 \cdot 2$ & $10 \cdot 3$ \\
\hline Sex male, $n$ and $\%$ & 149 & 50 & 77 & 41 \\
\hline BMI $\left(\mathrm{kg} / \mathrm{m}^{2}\right)$, mean and SD & $16 \cdot 7$ & 1.2 & $16 \cdot 6$ & 1.3 \\
\hline \multicolumn{5}{|l|}{ BMI category, $n$ and $\%$} \\
\hline$<16.0 \mathrm{~kg} / \mathrm{m}^{2}$ & 72 & 24 & 54 & 29 \\
\hline $16.0-16.99 \mathrm{~kg} / \mathrm{m}^{2}$ & 80 & 27 & 48 & 26 \\
\hline $17.0-18.49 \mathrm{~kg} / \mathrm{m}^{2}$ & 145 & 49 & 85 & 46 \\
\hline CD4 count (cells/ $\mu \mathrm{l})$, mean and SD & 156 & 96 & 114 & 99 \\
\hline Tuberculosis treatment pre-ART, $n$ and $\%$ & 82 & 28 & 43 & 23 \\
\hline Oedema at recruitment, $n$ and $\%$ & 8 & $2 \cdot 7$ & 4 & $2 \cdot 1$ \\
\hline \multicolumn{5}{|l|}{ Marital status, $n$ and $\%$} \\
\hline Married & 154 & 52 & 66 & 35 \\
\hline Widowed/widower & 32 & 11 & 26 & 14 \\
\hline Divorced & 52 & 18 & 72 & 39 \\
\hline Single - never married & 59 & 20 & 23 & 12 \\
\hline \multicolumn{5}{|l|}{ Occupation, $n$ and \% } \\
\hline Salaried employed & 61 & 21 & 6 & 3 \\
\hline Self-employed & 82 & 28 & 159 & 85 \\
\hline Housewife & 43 & 15 & 12 & 6 \\
\hline Student & 6 & 2 & 3 & 2 \\
\hline Unemployed & 105 & 35 & 7 & 4 \\
\hline \multicolumn{5}{|l|}{ Education, $n$ and $\%$} \\
\hline No education & 39 & 13 & 50 & 27 \\
\hline Primary (1-7 grades) & 153 & 52 & 123 & 66 \\
\hline Secondary (8-12 grades) & 88 & 30 & 13 & 7 \\
\hline Tertiary college/university & 17 & 6 & 1 & 0.5 \\
\hline
\end{tabular}

ART, antiretroviral therapy.

patients; therefore, further analyses included results from Tanzanians only. The varying time pre-ART influenced the shape of the appetite score curve over time, specifically because of the moving zero point of starting ART; Fig. 3 shows the results for Tanzanian patients with 14-20 d pre-ART. For all Tanzanians, on average appetite score was low at recruitment (overall: mean -1.07 for males, -0.97 for females), increased considerably during the pre-ART period, plateaued at the start of ART and then continued to increase more slowly throughout the trial (at 12 weeks overall: mean 0.93 for males, 0.94 for females). By 12 weeks of ART the range of responses had narrowed so that the vast majority of Tanzanian patients reported excellent or good appetite, moderate hunger, they could afford food and food tasted good or very good.

In the standardized porridge consumption test at recruitment, Tanzanian males ate a mean of $319 \mathrm{~g}$ porridge (95\% CI 297, $342 \mathrm{~g}, n$ 77), females $333 \mathrm{~g}$ (95\% CI 312, $353 \mathrm{~g}, n$ 110), Zambian males $267 \mathrm{~g}$ (95\% CI 254, $280 \mathrm{~g}$, $n$ 130) and females $246 \mathrm{~g}$ (95\% CI 235, $257 \mathrm{~g}, n$ 136). In Tanzania, by week 12 there was little change with males eating a mean of $328 \mathrm{~g}(95 \%$ CI 309, $348 \mathrm{~g}, n$ 77) and females $337 \mathrm{~g}$ (95\% CI 319, $355 \mathrm{~g}, n$ 110; Fig. 4). There was no difference in consumption between patients who had fasted prior to the test and those who had not fasted $(P=0 \cdot 17)$. The test was not conducted in Zambia at week 12 but in previous weeks little difference was shown across time points. Furthermore, in Lusaka, interim analysis of the results in June 2012 indicated that most intakes were clustered around $250 \mathrm{~g}$, the amount given in the first bowl, with few patients requesting additional bowls. Discussion with staff and patients suggested that patients liked the porridge but cultural norms prevented many from asking for multiple portions. From 19 June to 20 July 2012, we offered Zambian patients $400 \mathrm{~g}$, instead of $250 \mathrm{~g}$, porridge in the first bowl, reverting back to $250 \mathrm{~g}$ as in the protocol after 20 July. The mean amount consumed at recruitment increased for males to $334 \mathrm{~g}(95 \%$ CI $279,390 \mathrm{~g}, n$ 10) and for females to $317 \mathrm{~g}(95 \%$ CI 267, $366 \mathrm{~g}, n$ 14). The squares in Fig. 4(b) indicate results collected between 20 July and September 2012; most values over $250 \mathrm{~g}$ were collected during this period. Since the results appeared to reflect other factors than appetite, the outcome of interest, the porridge consumption test was stopped in Lusaka in September 2012.

\section{Associations of appetite with weight change for Tanzanian patients}

Change in appetite score over two weeks from the time points of recruitment, start of ART, and 2 and 6 weeks after starting ART was associated with the change in 
(a)

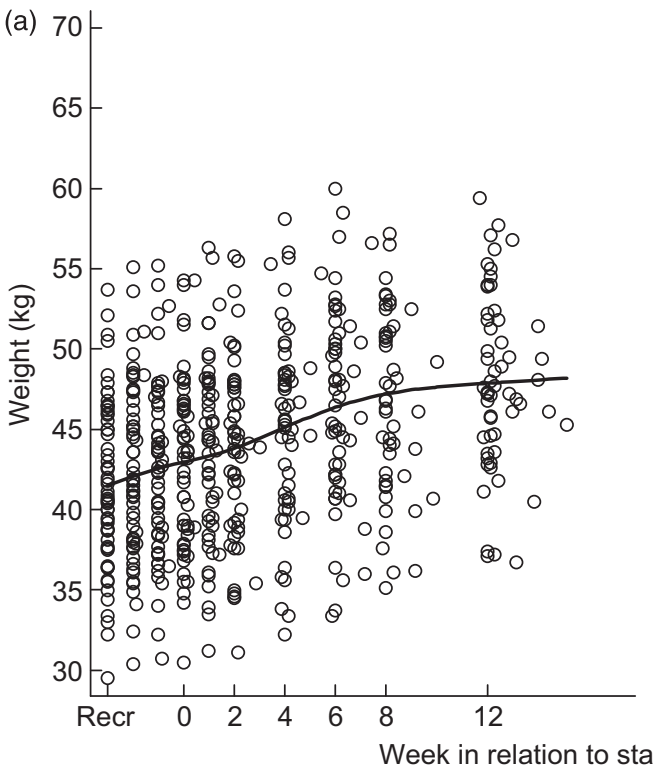

(c)

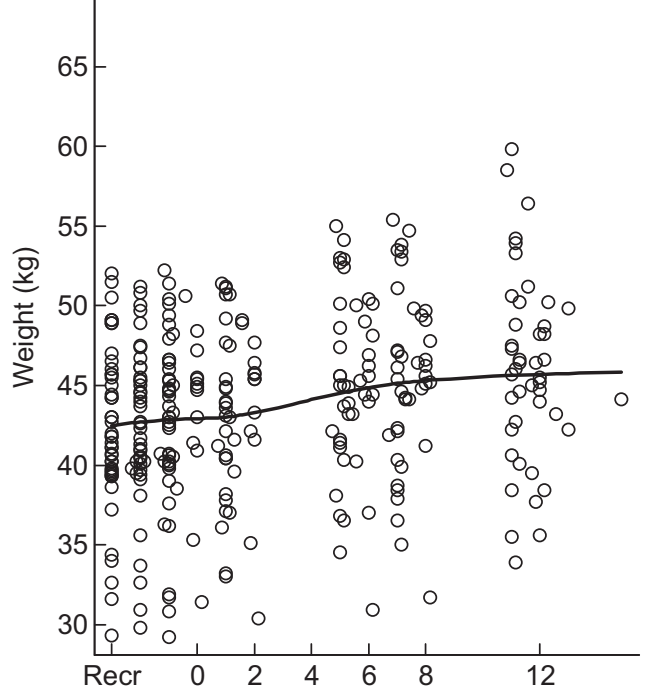

(b)

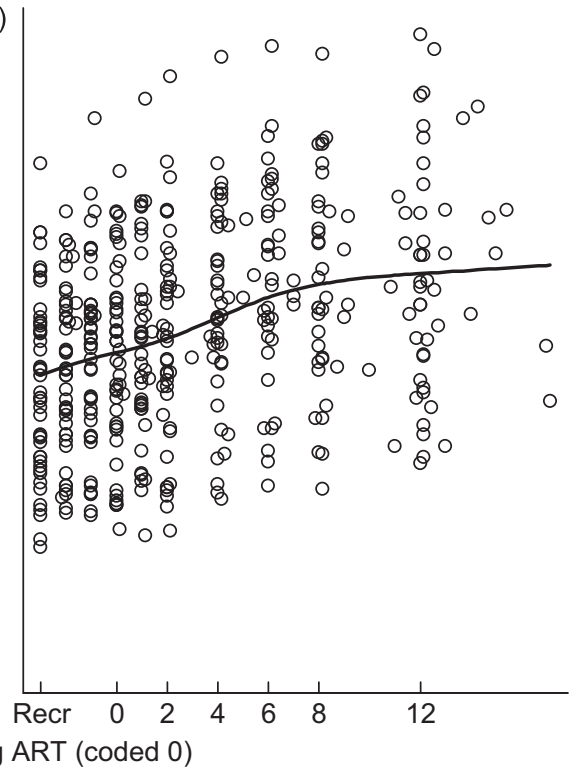

(d)

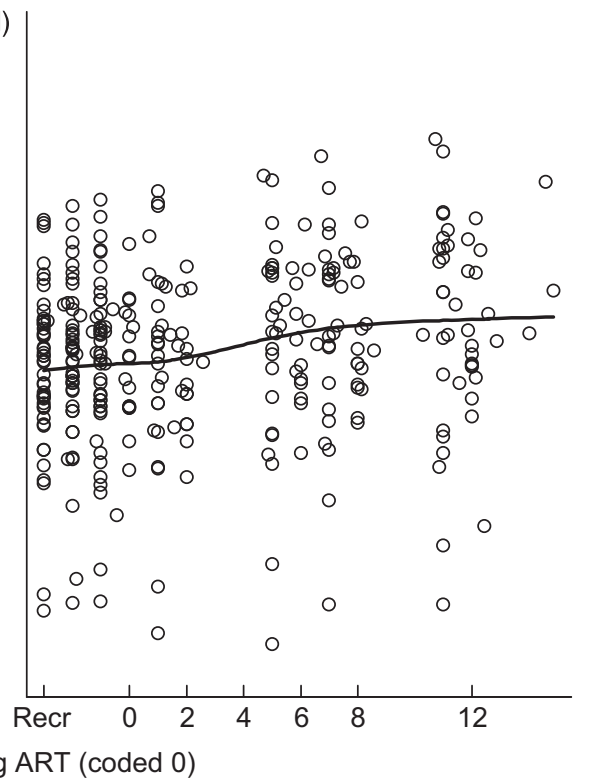

Fig. 2 Spline curves of participant weight over time for patients starting antiretroviral therapy (ART) 14-20 d after recruitment (Recr) for (a) fifty-seven Tanzanian women, (b) forty-six Tanzanian men, (c) forty-four Zambian women and (d) forty-seven Zambian men; Nutritional Support for Africans Starting Antiretroviral Therapy (NUSTART) trial

weight during the same two weeks (Table 3). The patients with an increase in appetite score (change $\geq 0 \cdot 25$ ) gained a mean of $41 \mathrm{~g}$ more weight daily than those whose appetite score decreased (change $\leq-0 \cdot 25$ ). There was also an association with overall changes in appetite score and weight from recruitment to 12 weeks of ART with a $26 \mathrm{~g} / \mathrm{d}$ difference in weight gain between those with increased or decreased appetite score. There was no evidence that hunger, measured as question \# 2 of the questionnaire, was associated with subsequent change in weight for the key two-week time periods. The porridge consumption test showed no association with weight in any of the above analyses.

\section{Discussion}

The aim of the present study was to compare appetite metrics suitable for use in low-income clinics or field situations to understand and monitor the nutritional rehabilitation and weight gain of malnourished HIVinfected adults starting ART. We estimated validity of the appetite measures by their association with weight change. Weight change indicates the quantity of food consumed in a population of free-living adults recovering from severe malnutrition and mostly without oedema at baseline, although we accept that weight changes can be due to hydration changes and that fat and lean tissue 


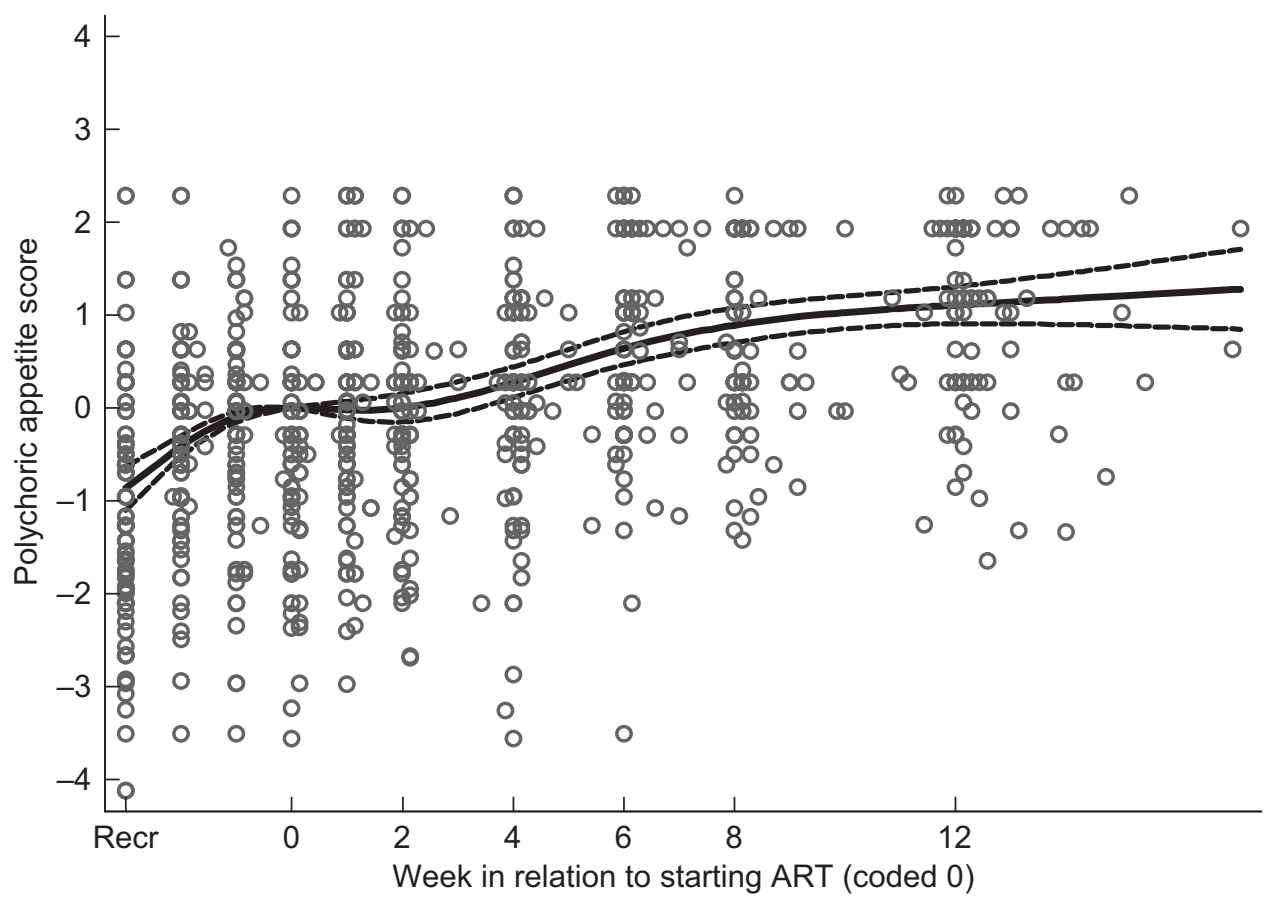

Fig. 3 Spline curve of appetite questionnaire score over time in 103 Tanzanian patients starting antiretroviral therapy (ART) 14-20 d after recruitment (Recr); Nutritional Support for Africans Starting Antiretroviral Therapy (NUSTART) trial. Lines - - - - - represent $95 \% \mathrm{Cl}$ and are zero at week 0 because the analysis used forced the curves through 0
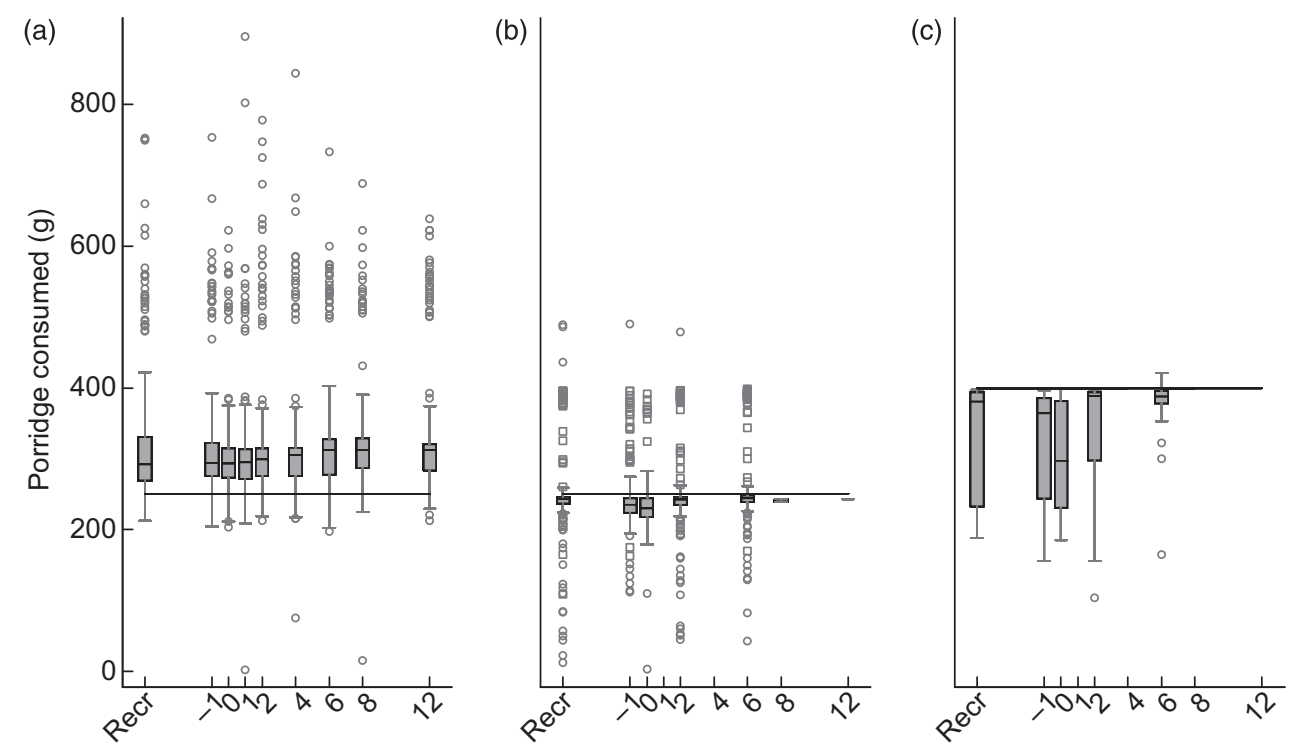

Week in relation to starting ART (coded 0)

Fig. 4 Box-and-whisker plots showing consumption of porridge over time by 187 Tanzanian patients (a) and 297 Zambian patients (b, c) starting antiretroviral therapy (ART) 14-20 d after recruitment (Recr); Nutritional Support for Africans Starting Antiretroviral Therapy (NUSTART) trial. Line inside the box represents the median; the bottom and top of the box represent the 25th and 75th percentile, respectively (i.e. the interquartile range); the whiskers indicate an additional $1.5 \times$ the interquartile range; and open circles represent outliers. Amount of porridge offered in the first bowl was $250 \mathrm{~g}$ in (a) and (b), $400 \mathrm{~g}$ in (c). In (b), squares denote tests after the $400 \mathrm{~g}$ test period in Zambia (July/August 2012) and before the decision to stop appetite testing in Zambia (September 2012)

deposited have different weights. Previous work with HIV-infected British adults showed that energy intake was the major factor driving weight changes ${ }^{(18)}$. However, a confounding issue due to our study design is that we provided more energy to patients from 2 to 6 weeks of ART and the results suggest that patients needed these food supplements to gain weight: comparison of Figs 2 and 3 shows that appetite increases preceded weight 
Table 3 Mean weight gain $(\mathrm{g} / \mathrm{d})$ and $95 \%$ confidence interval in relation to change in appetite for the same period in Tanzanian patients; Nutritional Support for Africans Starting Antiretroviral Therapy (NUSTART) trial

\begin{tabular}{|c|c|c|c|c|c|c|}
\hline \multirow[b]{2}{*}{ Time period and appetite measure } & \multicolumn{2}{|c|}{ Unadjusted } & \multicolumn{2}{|c|}{ Model 1} & \multicolumn{2}{|c|}{ Model 2} \\
\hline & $g / d$ & $95 \% \mathrm{Cl}$ & $g / d$ & $95 \% \mathrm{Cl}$ & $g / d$ & $95 \% \mathrm{Cl}$ \\
\hline \multicolumn{7}{|l|}{ Change in polychoric appetite score } \\
\hline \multicolumn{7}{|l|}{ Key two-week times } \\
\hline Decrease & 42 & 25,60 & 29 & 3,55 & 20 & $-21,61$ \\
\hline Little change & 70 & 46,94 & 58 & 28,88 & 49 & 6,92 \\
\hline Increase & 84 & 70,97 & 70 & 48,91 & 61 & 24,100 \\
\hline & \multicolumn{2}{|c|}{$P=0.001$} & \multicolumn{2}{|c|}{$P=0.001$} & \multicolumn{2}{|c|}{$P=0.002$} \\
\hline \multicolumn{7}{|l|}{ All follow-up } \\
\hline Decrease & 28 & 6,51 & 26 & 3,49 & 52 & 20,84 \\
\hline Little change & 50 & 28,70 & 45 & 23,67 & 62 & 34,91 \\
\hline Increase & 59 & 53,65 & 56 & 48,64 & 78 & 59,98 \\
\hline & \multicolumn{2}{|c|}{$P=0.03$} & \multicolumn{2}{|c|}{$P=0.03$} & \multicolumn{2}{|c|}{$P=0.05$} \\
\hline \multicolumn{7}{|l|}{ Change in appetite consumption test } \\
\hline \multicolumn{7}{|l|}{ Key two-week times } \\
\hline Decrease & 76 & 57,96 & 62 & 36,88 & 53 & 12,95 \\
\hline Little change & 66 & 52,80 & 51 & 28,74 & 41 & 0,82 \\
\hline \multirow[t]{2}{*}{ Increase } & 68 & 46,91 & 46 & 18,74 & 41 & $-1,84$ \\
\hline & \multicolumn{2}{|c|}{$P=0.69$} & \multicolumn{2}{|c|}{$P=0.52$} & \multicolumn{2}{|c|}{$P=0.61$} \\
\hline \multicolumn{7}{|l|}{ All follow-up } \\
\hline Decrease & 57 & 45,69 & 55 & 42,67 & 76 & 55,97 \\
\hline Little change & 56 & 46,65 & 52 & 41,64 & 77 & 54,101 \\
\hline \multirow[t]{2}{*}{ Increase } & 55 & 46,64 & 52 & 42,63 & 77 & 57,97 \\
\hline & \multicolumn{2}{|c|}{$P=0.98$} & \multicolumn{2}{|c|}{$P=0.94$} & \multicolumn{2}{|c|}{$P=0.98$} \\
\hline \multicolumn{7}{|c|}{ Hunger questionnaire at beginning of time period } \\
\hline \multicolumn{7}{|c|}{ Key two-week times } \\
\hline Not hungry & 106 & 55,157 & 95 & 45,146 & 84 & 23,145 \\
\hline Average hunger & 61 & 40,81 & 49 & 23,75 & 47 & 6,88 \\
\hline Moderately hungry & 71 & 57,86 & 64 & 38,90 & 57 & 17,98 \\
\hline \multirow[t]{2}{*}{ Very hungry } & 62 & 43,82 & 52 & 27,78 & 43 & 2,84 \\
\hline & \multicolumn{2}{|c|}{$P=0.36$} & \multicolumn{2}{|c|}{$P=0.28$} & \multicolumn{2}{|c|}{$P=0.40$} \\
\hline
\end{tabular}

ART, antiretroviral therapy.

Model 1 is adjusted for sex and, for the key two-week time data, also the key time point: recruitment, ART, ART +2 or ART +6 . Model 2 is adjusted for sex, key time point (if appropriate), baseline BMI group, baseline CD4 group, socio-economic status, oedema at baseline, tuberculosis diagnosis pre-ART. $P$ values are for the effect of the appetite variables in the linear regression models.

increases and a large proportion of the weight gain was during the period that more energy was provided.

Among Tanzanian patients, a short questionnaire was used to develop an overall population appetite score. This score was able to detect change in appetite that was associated with weight gain during the same two-week period or over the entire study. However, hunger score at the start of a two-week interval did not predict subsequent weight gain. The questionnaire could thus be used to understand appetite changes in the population but would not be useful clinically to detect patients who will respond well to nutritional therapy or who may need more intensive care or appetite stimulants.

A previous study conducted among malnourished HIVinfected adults starting ART in Lusaka measured appetite by a single question and compared it with dietary intake measured by $24 \mathrm{~h}$ dietary recall at recruitment and weeks 1, 4 and 12 after starting $\mathrm{ART}^{(19)}$. The self-reported appetite was associated with energy intake from dietary recall but it is difficult to assess energy intake by $24 \mathrm{~h}$ recall because of the need to assess portion size, and all intakes seemed low. Intake at baseline was not significantly associated with mortality to 12 weeks of ART but an increase in intake during this period was associated with increased survival. Our results provide comparable information that it is change in appetite - rather than appetite at a particular time, which may be heavily influenced by other patient characteristics and perceptions - that is more clinically meaningful.

The porridge consumption test of appetite, in addition to being more time-consuming and complex to carry out, did not vary over time in the study and was not associated with weight change. The test may actually be a better indicator of satiety, a feeling associated with the end of a meal, than with appetite, a feeling prior to eating. Ill patients may feel hungry but then find they are unable to eat much.

The spline curves of appetite score over time among Tanzanian patients showed complex changes of relevance to clinical care of such patients. There was a rapid increase in appetite between referral for ART and actually starting ART. During this interval patients were provided with treatments for opportunistic infections and other illnesses, counselling by medical staff and, for the intervention group, LNS containing high levels of vitamins and minerals; any of these could have resulted in improved appetite. 
Whether this resulted in a harmful refeeding syndrome cannot be determined from the data but the fact that increased diet intake in the previous Lusaka study was associated with increased survival ${ }^{(19)}$ suggests refeeding syndrome may not have been of major importance in this patient population. The start of ART was associated with a brief period when appetite plateaued, possibly a reaction to the drugs themselves. Although, likely for logistic reasons, food supplementation programmes often target patients starting $\operatorname{ART}^{(2,20)}$, it may be preferable to delay a few weeks until appetite is restored. Preliminary data from Ethiopia $^{(21)}$ showed that the timing of nutritional support with LNS affected its impact on lean mass and functional outcomes. Finally, the longer-term increases in appetite after several weeks of ART support evidence that appetite increases with ART and may reduce ART adherence among food-insecure patients ${ }^{(8)}$. These observations provide evidence for targeting nutritional support to malnourished or food-insecure patients after several weeks of ART.

A limitation of both appetite tests is that they provided reliable results in only one of the two study sites. Responses to the questionnaire were highly clustered in Zambia and likely reflected a combination of patients providing socially acceptable responses and nurses having trouble interpreting or explaining the questions; for example, some nurses interpreted the first question to mean usual appetite during the past few months of advanced HIV infection, not usual compared with healthy people. Part of the difficulty with the porridge consumption appetite test in Zambia appeared to be cultural in that people seemed willing to eat whatever amount of porridge was given, even when this was increased from $250 \mathrm{~g}$ to $400 \mathrm{~g}$, but not to ask for second bowls. Such cultural influences are likely stronger among adults than among young children fed by their mothers or even among primary school-aged children in whom similar tools have been used successfully in Africa ${ }^{(9,13)}$. In addition, there appeared to be some subtle and unrecorded aspect of the study nurses' behaviour in offering the porridge since there was an increase in requests for second bowls once the issue became a topic of discussion in the Zambian clinic. There may also have been differences in approach of the nurses in Tanzania from those in Zambia, even though all were following the same trial standard operating procedures. Our overall interpretation of a standardized meal as a test of appetite in adults is that it is too sensitive to minor differences in testers' attitudes, taste preferences of patients or to even the cultural differences between two cities in adjacent sub-Saharan African countries to be useful. However, the simple questionnaire, with additional training or minor modification, could likely be used successfully in Zambia as well as Tanzania.

An additional limitation of our study is that we remain blind to treatment allocation. The trial intervention products were two different food supplements, varying in vitamin and mineral content. A multiple micronutrient intervention increased appetite, measured by a standardized porridge intake test, among young HIV-infected South African children ${ }^{(12)}$ and Fe supplementation increased appetite, measured by both a standardized porridge intake test and a single question about appetite, among school-aged Kenyan children ${ }^{(13)}$. The intervention products may also have influenced the proportion lean or fat mass deposited, which would affect the association between appetite and weight change.

We conclude that a simple appetite questionnaire can be used to assess appetite among Tanzanian adults and could likely be adapted to work successfully for Zambian adults also. The questionnaire appeared not very useful as a clinical marker for individual patients, for example, of when to change from an initial stabilization phase in the management of malnutrition to provision of additional energy, since responses at a single time point did not predict subsequent weight change. However, the questionnaire may be useful for research and for guiding programmes; specifically, the results suggest that high-energy supplements for malnourished, HIV-infected adults should be targeted to patients who have been on ART several weeks when infections have been controlled and the appetite plateau at the start of ART has resolved.

\section{Acknowledgements}

Acknowledgements: The authors are grateful to the European and Developing Countries Clinical Trials Partnership for funding the study and to Nutriset, Malauney, France for preparing the lipid nutrient supplements. The work was conducted by the NUSTART study team which includes the following. Principal Investigator: Suzanne Filteau; Senior Investigators: Aase Bengaard Andersen, John Changalucha, Henrik Friis, Douglas Heimburger, Lackson Kasonka and Paul Kelly; statisticians and other senior research fellows: John Koethe, Daniela Manno, Natasha Larke, Andrea Rehman and Susannah Woodd; steering group: David Thurnham and Andrew Tomkins; Mwanza trial manager: George PrayGod; Lusaka trial managers: Molly Chisenga and Joshua Siame; Mwanza senior clinic team: Jeremiah Kidola, Michael Denna, Charles Masilingi, Eva Masesa, Elizabeth Fue and Neema Mpandachaloe; Lusaka senior clinic team: Anne Kanunga, Likando Munalula, Brenda Kapinda and Nellie Sikanyika; laboratory technicians: Julius Mngara, George Ogweno, Pius Ikigo, Mutinta Muchimba, Leo Beacroft and Celeste Gregg Smith; administrators and data managers: Yolanda Fernandez, Gunda Wandore, Aswile Jonas and Hildah Banda Mabuda. The authors are grateful also to nurses, data entry clerks, drivers and other support staff at both NUSTART sites. They thank the Data Safety and Monitoring Board - Nicholas Paton (chair), Kathy Baisley (statistician), Muhammad Bakari (Tanzanian representative), 
Sekelani Banda (Zambian representative) - for their support and advice, and the on-site monitors, Jim Todd and Max Katubulushi, for their assistance. Financial support: This work was supported by the European and Developing Countries Clinical Trials Partnership (grant \# IP.2009.33011.004); and the London School of Hygiene and Tropical Medicine (LSHTM; student travel grant to G.S.). The funders had no role in the design, analysis or writing of this article. Conflict of interest: None. Authorship: G.S. conducted a preliminary version of the research as part of her MSc studies at the LSHTM; M.C., J.S. and G.P. were responsible for the clinical work and contributed to G.S.'s MSc research; A.M.R. was responsible for statistical analyses and was assisted by S.W.; S.F. designed the study with assistance from P.K. and J.R.K.; S.F. drafted the manuscript; all authors contributed to the manuscript and approved the final version. Ethics of human subject participation: The study was approved by the ethics committees of the LSHTM, the University of Zambia and the National Institute for Medical Research, Tanzania.

\section{References}

1. Lawn SD, Harries AD, Anglaret $\mathrm{X}$ et al. (2008) Early mortality among adults accessing antiretroviral treatment programmes in sub-Saharan Africa. AIDS 22, 1897-1908.

2. Ndekha MJ, van Oosterhout JJ, Zijlstra EE et al. (2009) Supplementary feeding with either ready-to-use fortified spread or corn-soy blend in wasted adults starting antiretroviral therapy in Malawi: randomised, investigator blinded, controlled trial. BMJ 338, b1867.

3. Bowie C, Kalilani L, Marsh R et al. (2005) An assessment of food supplementation to chronically sick patients receiving home based care in Bangwe, Malawi: a descriptive study. Nutr J 4, 12.

4. Ashworth A, Khanum S, Jackson A et al. (2003) Guidelines for the inpatient treatment of severely malnourished children. http://www.who.int/nutrition/publications/severe malnutrition/9241546093/en/index.html (accessed June 2013).

5. Heikens GT, Bunn J, Amadi B et al. (2008) Case management of HIV-infected severely malnourished children: challenges in the area of highest prevalence. Lancet 371, 1305-1307.

6. Nyirenda C, Zulu I, Kabagambe E et al. (2009) Acute hypophosphataemia and hypokalaemia in a patient starting antiretroviral therapy in Zambia - a new context for refeeding syndrome? BMJ Case Reports doi:10.1136/ bcr.07.2008.0469; available at http://casereports.bmj.com/ cgi/content/abstract/2009/apr01_2/bcr0720080469
7. Nagata JM, Magerenge RO, Young SL et al. (2012) Social determinants, lived experiences, and consequences of household food insecurity among persons living with HIV/ AIDS on the shore of Lake Victoria, Kenya. AIDS Care 24, $728-736$.

8. Au JT, Kayitenkore K, Shutes E et al. (2006) Access to adequate nutrition is a major potential obstacle to antiretroviral adherence among HIV-infected individuals in Rwanda. AIDS 20, 2116-2118.

9. Dossa RA, Ategbo EA, van Raaij JM et al. (2002) An appropriate tool for appetite testing and evaluation in young children in Benin. Appetite 38, 99-109.

10. Kendall A, Olson CM \& Frongillo EA Jr (1995) Validation of the Radimer/Cornell measures of hunger and food insecurity. J Nutr 125, 2793-2801.

11. Wilson MM, Thomas DR, Rubenstein LZ et al. (2005) Appetite assessment: simple appetite questionnaire predicts weight loss in community-dwelling adults and nursing home residents. Am J Clin Nutr 82, 1074-1081.

12. Mda S, van Raaij JM, Macintyre UE et al. (2010) Improved appetite after multi-micronutrient supplementation for six months in HIV-infected South African children. Appetite $\mathbf{5 4}$ $150-155$.

13. Lawless JW, Latham MC, Stephenson LS et al. (1994) Iron supplementation improves appetite and growth in anemic Kenyan primary school children. J Nutr 124, 645-654.

14. Ulijaszek S \& Kerr D (1999) Anthropometric measurement error and the assessment of nutritional status. Br J Nutr $\mathbf{8 2}$, 165-177.

15. Kolenikov S \& Angeles G (2004) The use of discrete data in PCA: Theory, simulations, and application to socioeconomic indices. http://www.cpc.unc.edu/measure/ publications/wp-04-85 (accessed April 2014).

16. Harrell FE Jr (2001) Regression Modeling Strategies: With Applications to Linear Models, Logistic Regression, and Survival Analysis. New York: Springer.

17. Filmer D \& Pritchett L (2001) Estimating wealth effects without expenditure data - or tears: an application to educational enrolments in states of India. Demography $\mathbf{3 8}$, $115-132$

18. Macallan DC, Noble C, Baldwin C et al. (1995) Energy expenditure and wasting in human immunodeficiency virus infection. $N$ Engl J Med 333, 83-88.

19. Koethe JR, Blevins M, Bosire C et al. (2013) Self-reported dietary intake and appetite predict early treatment outcome among low-BMI adults initiating HIV treatment in subSaharan Africa. Public Health Nutr 16, 549-558.

20. Cantrell RA, Sinkala M, Megazinni K et al. (2008) A pilot study of food supplementation to improve adherence to antiretroviral therapy among food-insecure adults in Lusaka, Zambia. J Acquir Immune Defic Syndr 49, 190-195.

21. Olsen MF, Abdissa A, Kæstel P et al. (2014) Effects of nutritional supplementation for HIV patients initiating antiretroviral treatment: a randomised controlled trial in Ethiopia. BMJ (In the Press). 